\title{
Effects of pharmacological treatments on hippocampal NCAM1 and ERK2 expression in epileptic rats with cognitive dysfunction
}

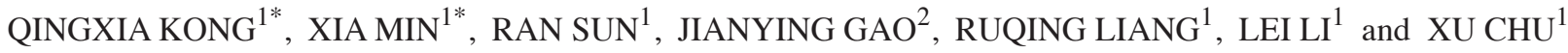 \\ Departments of ${ }^{1}$ Neurology and ${ }^{2}$ Nuclear Medicine, Affiliated Hospital of Jining Medical College, \\ Jining, Shandong 272129, P.R. China
}

Received November 28, 2015; Accepted November 3, 2016

DOI: $10.3892 / 01.2016 .4882$

\begin{abstract}
The present study aimed to investigate the effects of various pharmacological agents on the hippocampal expression of neural cell adhesion molecule 1 (NCAM1) and extracellular signal-regulated kinase 2 (ERK2) in epileptic rats with cognitive dysfunction. The experiments were conducted using 120 Wistar rats: 20 controls and 100 with pilocarpine-induced status epilepticus (SE). The SE rats were randomly assigned to 5 groups ( $n=20 /$ group) that received daily treatments for 1 month with one of the following: (i) saline (no effect on epilepsy); (ii) carbamazepine (an anticonvulsant); (iii) oxcarbazepine (an anticonvulsant); (iv) aniracetam (a nootropic); or (v) donepezil (an acetylcholinesterase inhibitor). Spatial learning and memory were assessed using a Morris Water Maze (MWM). Hippocampal tissue was assessed for NCAM1 and ERK2 messenger RNA (mRNA) expression by reverse transcription polymerase chain reaction, and protein expression by immunochemistry. The results revealed that SE rats had significantly poorer MWM performances compared with controls $(\mathrm{P}<0.01)$. Performance in $\mathrm{SE}$ rats was improved with donepezil treatment $(\mathrm{P}<0.01)$, but declined with carbamazepine $(\mathrm{P}<0.01)$. Compared with controls, saline-treated $\mathrm{SE}$ rats exhibited increased hippocampal NCAM1 mRNA expression $(\mathrm{P}<0.01)$. Among SE rats, NCAM1 mRNA expression was highest in those treated with donepezil, followed by aniracetam-, saline-, oxcarbazepine- and carbamazepine-treated rats. Compared to controls, saline-treated SE rats exhibited decreased hippocampal ERK2 mRNA expression $(\mathrm{P}<0.01)$. Among SE rats, ERK2 mRNA expression was highest in those treated with donepezil, followed by aniracetam, saline, oxcarbazepine and carbamazepine. NCAM1 and ERK2 protein expression levels were parallel to those of the mRNA.
\end{abstract}

Correspondence to: Dr Qingxia Kong, Department of Neurology, Affiliated Hospital of Jining Medical College, 89 Guhuai Road, Jining, Shandong 272129, P.R. China

E-mail: qingxiakong016@sohu.com

*Contributed equally

Key words: NCAM1, ERK2, hippocampus, pharmacological intervention, status epilepticus
In saline-treated SE rats, hippocampal ERK2 expression was decreased and NCAM1 expression was increased; thus, these two molecules may be involved in the impairment of spatial memory. Carbamazepine augmented this impairment, whereas donepezil was found to ameliorate the dysfunction associated with epilepsy. In conclusion, ERK2 and NCAM1 have significant roles in impairment of spatial memory in SE rats. Carbamazepine may increase this impairment, while donepezil may decrease this impairment.

\section{Introduction}

Epilepsy encompasses a number of different syndromes whose cardinal feature is a predisposition to recurrent, spontaneous seizure activity $(1,2)$. Although the specific mechanisms of these different syndromes may vary, at the cellular, neurophysiological level, a common theme is a loss of the normal balance between excitation and inhibition. As described by Scharfman (3) using temporal lobe epilepsy as an example, genes, developmental mechanisms and neuronal plasticity all serve functions in creating a state of hyperexcitability. Altered neuronal plasticity is central to the underlying pathophysiology of epilepsy.

Among the deficits associated with epilepsy are cognitive problems, particularly those related to memory impairment $(4,5)$. In status epilepticus (SE), which is defined as $\geq 30 \mathrm{~min}$ of continuous epileptic seizure activity, hippocampal-specific memory is a common and serious deficit. Liu et al (6) reported an association between abnormal place cells in the hippocampus and spatial memory in rats in which SE had been established. Other studies using rat epilepsy models have also demonstrated impaired spatial memory associated with neuronal remodeling in the hippocampus $(7,8)$.

With regard to neuronal plasticity, remodeling and cognition, the activities of neural cell adhesion molecules (NCAMs) and extracellular signal-regulated kinases (ERKs) appear to be interconnected (9-11). NCAMs are cell surface glycoproteins that mediate the recognition and adhesion of neural cells and contribute to neurogenesis and synaptic plasticity $(12,13)$. NCAM expression is altered in the hippocampus of epileptic rats $(14,15)$.

ERKs, including ERK1 and ERK2, also known as mitogen-activated protein kinases (MAPKs), are essential mediators of signal transduction pathways between cell 
surface receptors and the nucleus (16). ERKs are also involved in neurogenesis and synaptic plasticity (17). As with NCAMs, studies on rodent models of epilepsy have revealed altered ERK expression and activity in the hippocampus $(16,18,19)$. In addition, during neurogenesis, NCAM expression is dependent on ERK signaling (20). Thus, the expression and activity of NCAM and ERK are interdependent under normal and neuropathological conditions. However, their complex interactions are not fully understood.

From a clinical perspective, one issue associated with epileptogenesis and impaired cognition is how best to treat these disorders. Although the number of pharmaceutical agents available to treat these disorders has increased in recent decades (21), there remain significant problems associated with their use due to differences in their clinical efficacy and, in particular, unwanted side effects (22). New generation drugs may be less toxic or better tolerated by patients with epilepsy; however, they may also exacerbate associated conditions, particularly cognitive impairments, for example, topiramate may cause cognitive impairment and have effects on attention, semantic functioning and language $(23,24)$. Furthermore, little is known regarding the effects of these drugs on the underlying molecular pathways that are associated with epileptogenesis and impaired cognition.

The current preliminary investigation sought to determine the effects that various classes of pharmacological agents may have on NCAM1 and ERK2 expression at the messenger RNA (mRNA) and protein levels in the hippocampal tissues of SE rats with spatial memory impairment. For this purpose, a pilocarpine-induced model of SE in rats was utilized, as this reflects a number of the neurophysiological and cognitive alterations observed in human epilepsy $(25,26)$. Changes in spatial memory were evaluated using a Morris Water Maze (MWM), and the effects of four commonly used drugs were assessed: Two anticonvulsants (carbamazepine and oxcarbazepine); and two drugs used to treat cognitive impairments, aniracetam (a nootropic) and donepezil (an acetylcholinesterase inhibitor). The results indicate that these anticonvulsants may aggravate impaired spatial memory, whereas the cognition enhancers may improve spatial memory, and these differential pharmacological effects on memory task performance are associated with the differential effects on NCAM1/ERK2 expression in the hippocampus.

\section{Materials and methods}

Animals and experimental groups. A total of 120 healthy, adult male Wistar rats (6 weeks of age; body weight, 180-200 g) were provided by Shandong Lukang Record Pharmaceutical Co., Ltd. (Jining, China). Rats were exposed to a $12 \mathrm{~h}$ light/12 h dark cycle and raised in separate cages in a conventional environment with free access to food and water. Rats were randomly divided into a control group $(n=20)$ and an experimental group $(n=100)$ in which SE was established (model described in subsequent paragraph). The experimental group was randomly divided into five groups (n=20/group) as follows: i) an epileptic (no pharmacological treatment) group; and groups treated with ii) carbamazepine (Beijing Nuohua Pharmaceutical Co., Ltd., Beijing, China), iii) oxcarbazepine (Sihuan Pharmaceutical Holdings Group Ltd., Beijing, China), iv) aniracetam (Shanghai Pharmaceuticals Holding Co., Ltd., Shanghai, China), or v) donepezil (Eisai China, Inc., Shanghai, China) (treatments described below). This study was approved by the Ethics Committee of Jining Medical College (Jining, China).

Epilepsy model and pharmacological treatments. Established methods for generating SE in rats were used $(25,26)$. First, lithium chloride (Sigma-Aldrich, St. Louis, MO, USA) was diluted in sterile saline and administered to each rat by intraperitoneal (i.p.) injection at a dose of $127 \mathrm{mg} / \mathrm{kg}$. After 18-24 h, methyl scopolamine $(1 \mathrm{mg} / \mathrm{kg}$ in sterile saline; MedChem Express, Princeton, NJ, USA) was administered by i.p. injection to dampen cholinergic responses in the periphery. After $30 \mathrm{~min}$, pilocarpine (Beijing Zhongshan Jinqiao Biotechnology Co., Ltd., Beijing, China) at $10 \mathrm{mg} / \mathrm{kg}$ in sterile saline was administered by i.p. injection. After a further $20 \mathrm{~min}$, seizure activity was monitored with a video camera (DS126311; Canon Inc., Tokyo, Japan). If no seizure activity of Racine grades IV-V appeared within $30 \mathrm{~min}$ (grades defined below), pilocarpine administration was continued at 30-min intervals until SE was established for $\geq 30$ min. Diazepam (Beijing Shuanghe Pharmaceutical Co., Ltd., Beijing, China) at $10 \mathrm{ml} / \mathrm{kg}$ was used to terminate the attack following 4-h SE.

After the SE model was successfully established in each group of mice (except for the untreated controls), the experimental drugs or saline (for untreated SE rats; 'epileptic rats') were administered. The experimental drugs were dissolved in saline and administered by oral gavage within a 24 -h period each day. Pharmaceutical drugs or saline were given for a period of one month after the SE model was established. Carbamazepine and oxcarbazepine were administered at $200 \mathrm{mg} / \mathrm{kg}$; aniracetam was administered at $10 \mathrm{mg} / \mathrm{kg}$; and donepezil was administered at $1 \mathrm{mg} / \mathrm{kg}$; all drug dosages were selected based on the manufacturer's protocol. The non-pharmacologically treated group was administered saline at $10 \mathrm{ml} / \mathrm{kg}$.

\section{Behavioral observations}

Seizure grading. Based on Racine's grading system (27), seizures were classified into one of five grades: I, mouth and facial movements (wet-doggish chattering, convulsive tics, chewing); II, rhythmic head nodding; III, forelimb clonus (spasm in one forelimb); IV, rearing with forelimb clonus (spasms in bilateral forelimbs, one limb standing); or V, rearing and falling with forelimb clonus (generalized motor convulsions, trunk imbalance, falling, myoclonus). When seizures appeared consecutively with no normal activities (grades IV-V), this was considered SE and indicated that the model was successfully induced. After SE had been established and pharmacological intervention was completed, spatial orientation and memory tests were conducted using an MWM (28).

Task setting. The MWM was a round pool (diameter, $1.2 \mathrm{~m}$ ) divided into four quadrants. The water temperature within the pool was maintained at $20-22^{\circ} \mathrm{C}$. For certain tests, a circular platform (diameter, $12 \mathrm{~cm}$ ) was fixed in place in one quadrant with its surface at $1 \mathrm{~cm}$ above the water level. Before tests were conducted, each rat was allowed to train by swimming freely for two 2-min periods, one in the morning and one in the afternoon. 
Spatial navigation learning. An origin was set at a fixed point in one of the four quadrants, and the platform was the end point. Each rat was placed into the pool facing the pool wall. The time taken for the rat to swim from the origin to the end point was recorded as the escape (latency) period. Each experiment allowed a maximum latency period of 120 $\mathrm{sec}$; if a rat did not locate the platform after $120 \mathrm{sec}$, it was guided to the platform to rest for $30 \mathrm{sec}$ and the latency period was recorded as $120 \mathrm{sec}$. For all groups, each rat entered the water one time from each of the four quadrants, and the mean latency time was recorded each day. Rats were tested for five consecutive days at a fixed time each day.

Probe trials for spatial memory. For these trials, the platform was removed. A rat was placed in the platform quadrant and allowed to swim. Over a $120 \mathrm{sec}$ period, the amount of time spent in each quadrant, including the 'platform quadrant' was recorded. These results were expressed as the percentage (\%) of time spent in each quadrant.

Data acquisition. The behaviors of the rats in the MWM were videotaped, and data was processing using a MWM real-time processing system that was devised in-house.

Brain specimen processing. After MWM testing was completed, rats were anesthetized with $10 \%$ chloral hydrate (200 mg/kg; Shanghai Pharmaceuticals Holding Co., Ltd.) by i.p. injection. The brains were processed in two ways for each group of rats. For one sub-group, following anesthesia, the chest was opened to expose the heart. A syringe needle was inserted into the left ventricle, the right atrial appendage was cut, and saline $\left(4^{\circ} \mathrm{C}\right)$ was rapidly infused until there was an outflow of clear liquid from the right atrial appendage. Subsequently, 4\% paraformaldehyde (Beijing Dingguo Changsheng Biotechnology Co., Ltd., Beijing, China) was perfused at $10 \mathrm{ml} / \mathrm{min}$ until the limbs were rigid, confirming sacrifice. Following removal of the brain, the hippocampus was identified and removed, and then fixed in $4 \%$ formaldehyde for $24 \mathrm{~h}$. The tissue was dehydrated with an ethanol series and xylene, and embedded in paraffin, before serial sections were cut at $40-\mu \mathrm{m}$ thickness (left and right sides). These specimens were used for immunostaining, as described subsequently. For the other sub-group, rats were anesthetized and decapitated, and the skull was rapidly cut open. The hippocampus was removed, rapidly frozen in liquid nitrogen, and stored at $-20^{\circ} \mathrm{C}$. These specimens were used for the reverse transcription-polymerase chain reaction (RT-PCR) analysis as described.

RT-PCR analysis of NCAM1 and ERK2 mRNA expression in rat hippocampus. NCAM1 and ERK2 mRNA levels were detected by RT-PCR using the frozen stored hippocampal tissue from five rats in each group. Total RNA from 0.5-1 g of tissue was extracted using RNeasy Mini Kits (Qiagen, Beijing, China) according to the manufacturer's instructions. cDNA was synthesized using a reverse transcription kit (Invitrogen; Thermo Fisher Scientific, Beijing, China) according to the manufacturer's instructions. The cDNA product was used for RT-PCR. Primers were designed by Sangon Biotech Co., Ltd. (Shanghai, China), and the sequences were as follows: ERK2 forward, 5'-CTTGAAGACACAGCACCTCAG-3', and reverse, 5'-CTTTGGAGTCAGCGTTTGGG-3'; NCAM1 forward, 5'-CAAAAATGACGAAGCCGAAT-3', and reverse, 5'-GTG
GACGTTCTCCAGGTGAT-3'; $\beta$-actin (internal reference) forward, 5'-GCCATGTACGTAGCCATCCA-3', and reverse, 5'-GAACCGCTCATTGCCGATAG-3'.

PCR amplification was performed using a GeneAmp ${ }^{\circledR}$ PCR System 9700 (Applied Biosystems; Thermo Fisher Scientific). RT-PCR reaction mixtures $(25 \mu \mathrm{l}$ total) included $0.5 \mu \mathrm{l}$ of cDNA, $0.5 \mu \mathrm{l}$ of each primer (forward and reverse), $11 \mu \mathrm{l}$ of $\mathrm{ddH}_{2} \mathrm{O}$, and $12.5 \mu \mathrm{l}$ of SYBR Green Supermix (Takara Bio, Inc., Otsu, Japan). PCR amplification conditions were an initial denaturation at $94^{\circ} \mathrm{C}$ for $3 \mathrm{~min}$, followed by cycles of $94^{\circ} \mathrm{C}$ for $50 \mathrm{sec}, 56^{\circ} \mathrm{C}$ for $50 \mathrm{sec}$, and $72^{\circ} \mathrm{C}$ for $50 \mathrm{sec}(33 \mathrm{cycles}$ for NCAM1; 32 cycles for ERK2). Finally, $5 \mu 1$ of each amplified PCR product was separated by electrophoresis on a $1 \%$ agarose gel. Separated bands in the gel were photographed using a gel imaging system (GelDoc XR+, Bio-Rad Laboratories, Inc., Hercules, CA, USA). Quantitative analysis was conducted using Gel-Pro Analyzer software (Media Cybernetics, Rockville, MD, USA). Target gene mRNA expression was normalized to that of $\beta$-actin, which was used as the internal reference.

Immunohistochemical analysis of NCAM1 and ERK2 protein expression in rat hippocampus. Paraffin-embedded hippocampus tissue sections from 10 rats in each group $(5$ sections for NCAM1 staining and 5 sections for ERK2 staining) were deparaffinized, rehydrated and treated with citric acid. Sections were immersed in a $3 \% \mathrm{H}_{2} \mathrm{O}_{2}$ solution for $10 \mathrm{~min}$ to block endogenous peroxidase activity, and then rinsed three times ( 2 min each) in phosphate-buffered saline (PBS).

Sections were incubated with either mouse anti-human monoclonal anti-NCAM1 (catalog no., ab9272; Abcam, Cambridge, UK) or mouse anti-rat monoclonal anti-ERK2 (catalog no., sc-154; Beijing Zhongshan Jinqiao Biotechnology Co., Ltd.) (diluted 1:200 in PBS with $0.1 \%$ bovine serum albumin) antibodies at $37^{\circ} \mathrm{C}$ for $1-2 \mathrm{~h}$. As a negative control, an isotype matched immunoglobulin (Wuhan Amyjet Scientific Co., Ltd., Wuhan, China) was used in place of the primary antibody. After incubation, the sections were rinsed 3 times ( 2 min each) with PBS. A secondary antibody (HRP-conjugated goat anti-mouse IgG SABC kit; catalog no., SA1027; Boster Biological Technology, Pleasanton, CA, USA) was added and incubated at room temperature or $37^{\circ} \mathrm{C}$ for $20 \mathrm{~min}$, following by 3 rinses ( 2 min each) with PBS. Finally, a 3,3'-diaminobenzidine solution was added for color development. After washing, the slides were mounted and viewed under a light microscope (BX50; Olympus Corp., Tokyo, Japan).

The number of positive cells in five randomly selected fields (x400 magnification) was counted and recorded as the percentage (\%) of positive cells from the total number of cells counted. NCAM1-positive cells were stained brown, and ERK2 positive cells were stained yellowish-brown.

Statistical analysis. RT-PCR results were analyzed using Microsoft Office Excel ${ }^{\circledR} 2010$ software (Microsoft, Inc., Redmond, WA, USA). Results for spatial navigation tests were compared by analysis of variance (ANOVA) by accounting for group and time (experimental day) effects. Group spatial memory probe test results were compared by ANOVA. Group results for RT-PCR and immunohistochemical staining results were compared by t-tests. Statistical analysis used SPSS 


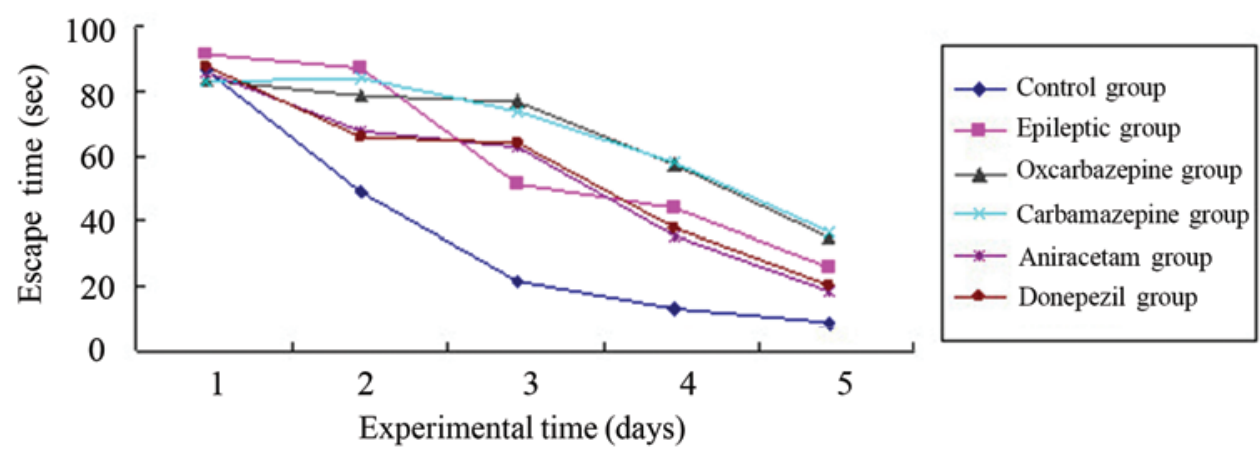

Figure 1. Morris water maze escape times. Results are the mean escape (latency) times (in seconds) for each group per experimental day ( $\mathrm{n}=20$ rats/group). Each rat was tested on 5 consecutive days at the same time each day.

version 13.0 (SPSS, Inc., Chicago, IL, USA). P<0.05 was considered to indicate a statistically significant difference.

\section{Results}

Spatial navigation learning. A total of 20 rats comprised a control group for normal behavioral observations, and SE was successfully established in 100 rats. Following pharmaceutical treatments, an MWM was used to test each rat for spatial navigation learning on 5 successive days. Figure 1 shows the mean escape (latency) times for each group of rats per day. As expected, the normal (control) group adapted well to this task, with the group's mean latency time decreasing each day for 5 days. By contrast, all groups of rats in which SE was established had a significantly longer mean latency time compared with the control group, even after 5 days $(\mathrm{P}=0.002$ compared to control group). Thus, SE significantly altered memory associated with the spatial navigation learning task in the rats.

Among the experimental (SE) rat groups, the time-course learning curves for this task were nearly identical for the saline-treated 'epileptic' group and the groups treated with aniracetam and donepezil. Thus, these agents used for cognitive impairment did not improve the SE-associated memory impairment. In addition, the time-course learning curves indicated significantly poorer memory in the groups treated with the anticonvulsants carbamazepine and oxcarbazepine ( $\mathrm{P}=0.001$ vs. saline-treated $\mathrm{SE}$ group).

Probe trials for spatial memory. The MWM was also used for probe trials for spatial memory following the removal of the escape platform from the pool. Table I shows the mean percentages of time that rats in each group spent swimming in each pool quadrant over a $120 \mathrm{sec}$ period (one trial per rat). Quadrant IV was the 'platform quadrant'. Normal control rats had the best retained spatial memory as demonstrated by spending the longest time swimming in quadrant IV (mean, $50.62 \%$ of swimming time). By contrast, all groups of rats in which SE was established spent significantly less time in quadrant IV than control rats $(<50 \%$ of swimming time; $\mathrm{P}<0.01)$.

With regard to pharmacological intervention in the SE rats, compared to the 'epileptic' group (saline treatment; mean, $36.65 \%$ of swimming time in quadrant IV), rats treated with donepezil had significantly better spatial memory (mean, $46.65 \pm 13.28 \%$ of swimming time in quadrant IV; $P=0.007$ ), whereas rats treated with carbamazepine had significantly worse spatial memory (mean, $26.84 \pm 8.68 \%$ of swimming time in quadrant IV; $\mathrm{P}=0.005)$. Other drugs did not demonstrate a significant difference in their effect on spatial memory compared with that of saline treatment.

Thus, based on the two MWM tasks, the current rat SE model exhibited significant hippocampus-associated spatial learning and memory impairments, and the different classes of pharmacological agents investigated had varying effects on these impairments (Table I).

Hippocampal expression of NCAM1 mRNA. RT-PCR analysis of NCAM1 and $\beta$-actin mRNA expression was conducted in frozen hippocampal tissue from each group of rats. Figure 2 shows the mean relative NCAM1 expression (NCAM1/ $\beta$-actin mRNA ratios) for each group (5 rats/group). The mean NCAM1 mRNA expression was significantly higher in the epileptic group (saline treatment) than in the normal control group $(\mathrm{P}<0.01)$. Thus, the SE model resulted in increased NCAM1 mRNA expression in the hippocampus.

Among SE rats subjected to pharmacological intervention, those treated with carbamazepine had significantly lower relative NCAM1 mRNA expression compared to the epileptic group $(\mathrm{P}=0.005)$, whereas rats treated with donepezil had significantly higher relative NCAM1 mRNA expression compared to the epileptic group ( $\mathrm{P}=0.001)$. Thus, among $\mathrm{SE}$ rats, a drug used to treat cognitive impairment resulted in relatively higher NCAM1 mRNA expression in the hippocampus, whereas an anticonvulsant agent resulted in relatively lower NCAM1 mRNA expression.

In SE rats, relative NCAM1 mRNA expression in the hippocampus was observed to be highest in those treated with donepezil, followed by those treated with aniracetam, saline, oxcarbazepine and carbamazepine (Table II).

Hippocampal expression of ERK2 mRNA. Frozen hippocampal tissue from each group of rats was also used for RT-PCR analysis of ERK 2 mRNA expression. Figure 3 shows the mean relative ERK2 expression (ERK2/ $\beta$-actin mRNA ratios) results per group (5 rats/group). In contrast to NCAM1 mRNA expression, the mean relative ERK2 mRNA expression was significantly lower in the epileptic group (saline treatment) than in the normal control group $(\mathrm{P}=0.005)$. Thus, the SE model resulted in decreased ERK2 mRNA expression in the hippocampus. 
Table I. Results of Morris Water Maze tasks in rats of each group ( $n=20 /$ group).

\begin{tabular}{|c|c|c|c|c|c|c|c|}
\hline \multirow[b]{2}{*}{ Group } & \multirow[b]{2}{*}{ Group number } & \multicolumn{3}{|c|}{ Escape (latency) } & \multicolumn{3}{|c|}{ Swimming in 'platform quadrant' } \\
\hline & & Time (sec; mean \pm SD) & P-value & P-value ${ }^{b}$ & Time $(\% ;$ mean $\pm \mathrm{SD})$ & P-value ${ }^{a}$ & $\mathrm{P}$-value \\
\hline Control & 1 & $35.78 \pm 4.84$ & - & 0.002 & $50.62 \pm 11.47$ & - & 0.004 \\
\hline Epileptic & 2 & $67.14 \pm 7.37$ & 0.002 & - & $36.65 \pm 13.28$ & 0.004 & - \\
\hline Carbamazepine & 3 & $81.23 \pm 9.46$ & $<0.001$ & 0.001 & $26.84 \pm 08.68$ & 0.001 & 0.005 \\
\hline Oxcarbazepine & 4 & $71.59 \pm 8.81$ & $<0.001$ & 0.241 & $31.55 \pm 14.13$ & 0.002 & 0.153 \\
\hline Aniracetam & 5 & $68.96 \pm 6.73$ & 0.001 & 0.630 & $41.55 \pm 11.17$ & 0.026 & 0.009 \\
\hline Donepezil & 6 & $53.75 \pm 6.74$ & 0.031 & 0.001 & $46.65 \pm 10.38$ & 0.529 & 0.007 \\
\hline
\end{tabular}

${ }^{\mathrm{a}} \mathrm{vs}$. control group; ${ }^{\mathrm{b}} \mathrm{vs}$. epileptic group. Analysis of variance was used to compare the differences between groups. SD, standard deviation.

A

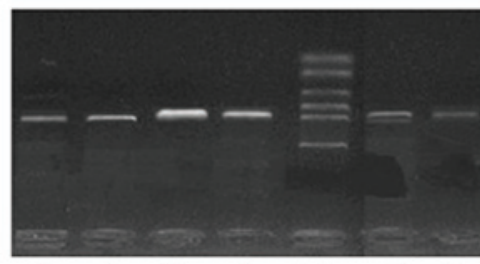

$\begin{array}{lllllll}1 & 2 & 3 & 4 & \mathrm{~m} & 5 & 6\end{array}$
$\mathbf{B}$

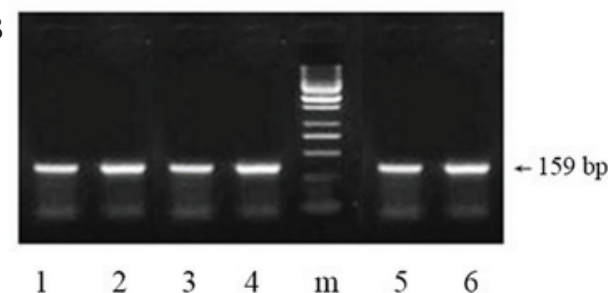

1 Oxcarbazepine; 2 Epileptic; 3 Donepezil; 4:Aniracetam; 5 Carbamazepine; 6 control; m marker

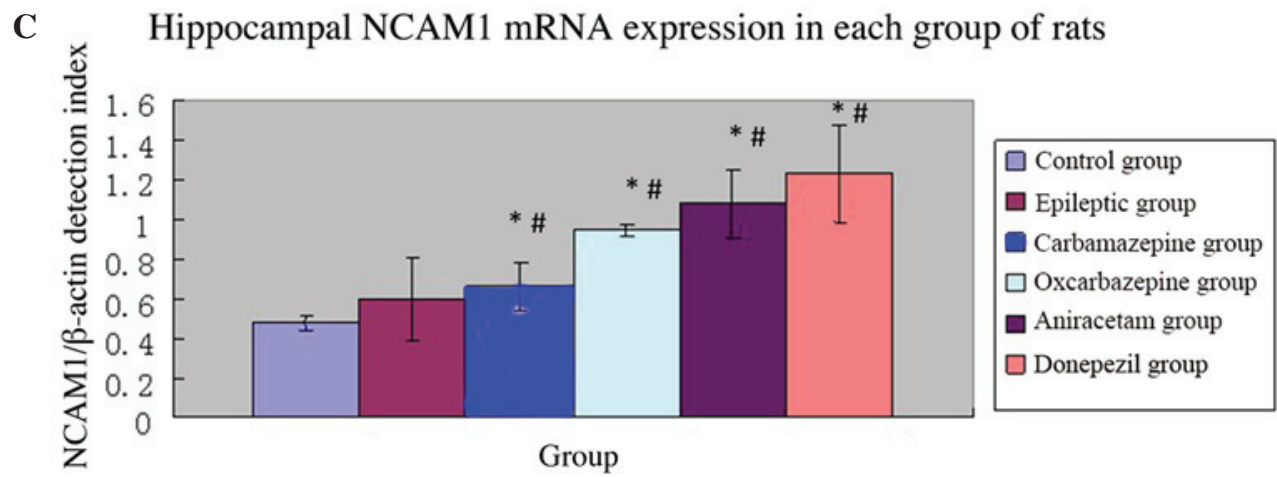

Figure 2. Relative hippocampal tissue expression of NCAM1 mRNA. Results for each group of rats are the NCAM1/ 3 -actin mRNA ratios (n=5 rats/group) Agarose gel electrophoresis of (A) NCAM1 and (B) $\beta$-actin. (C) Relative hippocampal tissue expression of NCAM1 mRNA. "P<0.01 vs. epileptic group; ${ }^{\#} \mathrm{P}<0.01$ vs. control group. NCAM1, neural cell adhesion molecule 1 ; mRNA, messenger RNA.

In SE rats subjected to pharmacological intervention, those treated with carbamazepine had significantly lower relative ERK2 mRNA expression compared with the epileptic group $(\mathrm{P}=0.005)$. However, rats treated with donepezil had significantly higher relative ERK2 mRNA expression compared to the epileptic group $(\mathrm{P}=0.005)$. Thus, among $\mathrm{SE}$ rats, drugs used for the treatment of cognitive impairment resulted in relatively higher ERK2 mRNA expression in the hippocampus, whereas treatment with anticonvulsant agents resulted in relatively lower ERK2 mRNA expression in the hippocampus.

As with NCAM1 mRNA expression, the relative ERK2 mRNA expression was highest in the hippocampus of SE rats treated with donepezil, followed by aniracetam, saline, oxcarbazepine and carbamazepine.

Regarding the trends in NCAM1 and ERK2 mRNA expression, compared to normal rats, saline-treated SE rats had relatively higher NCAM1 mRNA and relatively lower ERK2 mRNA expression in the hippocampus. Among SE rats subjected to pharmacological intervention, compared with saline-treated SE rats, those treated with anticonvulsants (carbamazepine and oxcarbazepine) had relatively lower NCAM1 and ERK2 mRNA expression in the hippocampus, whereas rats treated with drugs to treat cognitive impairment (donepezil and aniracetam) had relatively higher NCAM1 and ERK2 mRNA expression in the hippocampus (Table II).

Hippocampal expression of ERK2 and NCMA1 protein. Figure 4 shows the results of the immunohistochemical analysis of NCMA1 and ERK2 protein expression in hippocampal tissue. Similar to the mRNA results, saline-treated SE rats had a greater percentage of NCAM1-positive cells in the hippocampus compared to control rats $(\mathrm{P}=0.001)$. Among the groups of $\mathrm{SE}$ rats, 
A

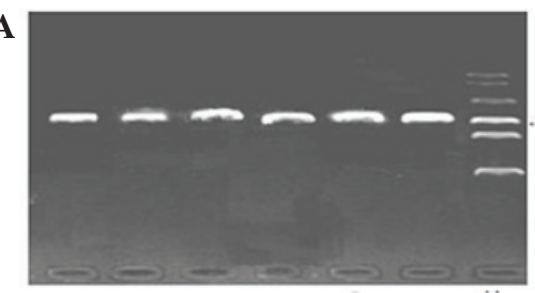

$\begin{array}{lllllll}1 & 2 & 3 & 4 & 5 & 6 & \mathrm{~m}\end{array}$

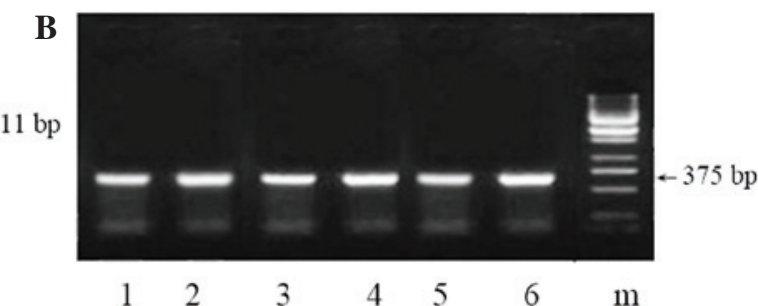

$\begin{array}{lllllll}1 & 2 & 3 & 4 & 5 & 6 & \mathrm{~m}\end{array}$

1 Oxcarbazepine; 2 Epileptic; 3 Donepezil; 4:Aniracetam; 5 Carbamazepine; 6 control; m marker

C

Hippocampal ERK2 mRNA expression of rats in each group

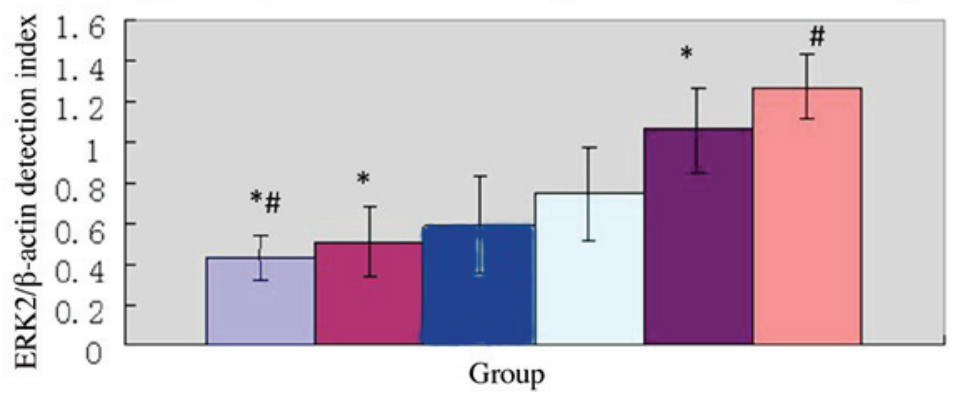

$\square$ Carbamazepine group

$\square$ Oxcarbazepin group

Epileptic group

$\square$ Control group

$\square$ Aniracetam group

$\square$ Donepezil group

Figure 3. Relative hippocampus tissue expressions of ERK mRNA. Results for each group of rats are the ERK/ $\beta$-actin mRNA ratios ( $\mathrm{n}=5$ rats/group). Agarose gel electrophoresis of (A) ERK and (B) $\beta$-actin. (C) Relative hippocampal tissue expression of ERK mRNA. ${ }^{\text {P }}<0.01$ vs. epileptic group; ${ }^{\text {P }}<0.01$ vs. control group. ERK2, extracellular signal-regulated kinase 2; mRNA, messenger RNA.

NCAM1- and ERK2-positive neurons in the hippocampus for each group

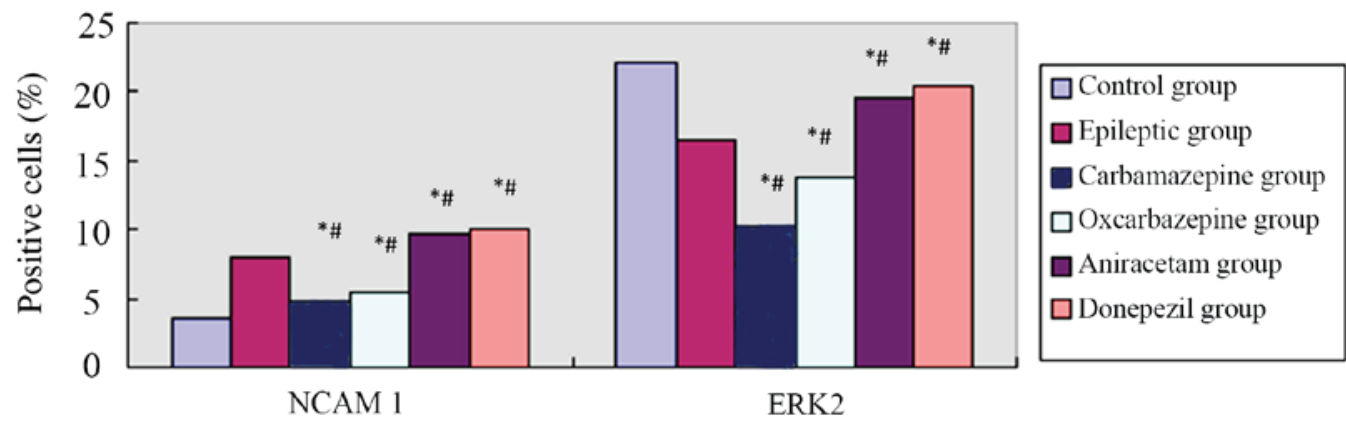

Figure 4. Hippocampus tissue expressions of ERK2 and NCAM proteins. Results for each group of rats ( $\mathrm{n}=5$ rats/group) are the percent of hippocampus cells positive for NCAM1 and ERK. Percentages are the $\%$ positive cells in 5 randomly selected microscopic fields (400x magnification). ${ }^{*}<0.01$ vs. epileptic roup; ${ }^{\text {"P }}<0.01$ vs. control group. NCAM1, neural cell adhesion molecule 1; ERK2, extracellular signal-regulated kinase 2.

the percentages of NCAM1-positive cells in the hippocampus were ordered high to low as follows: Donepezil, aniracetam, saline, oxcarbazepine and carbamazepine. Furthermore, as with the mRNA results, saline-treated SE rats had relatively fewer ERK2-positive cells in the hippocampus compared to control rats $(\mathrm{P}=0.003)$. Among the groups of $\mathrm{SE}$ rats, the percentages of ERK2-positive cells in the hippocampus were ordered high to low as follows: Donepezil, aniracetam, saline, oxcarbazepine and carbamazepine. Thus, the effects of pharmacological intervention on NCAM1 and ERK2 expression in the hippocampus of epileptic rats with cognitive dysfunction was confirmed at the mRNA and protein levels (Table III).

\section{Discussion}

The purpose of the present preliminary investigation was to assess the effects of various classes of pharmacological agents on cognitive dysfunction (spatial memory impairment) in a pilocarpine-induced rat model of status epilepticus (SE), as this model reflects some of the neurophysiological and cognitive deficits observed in human epilepsy $(25,26)$. Using Morris Water Maze (MWM) trials, the SE rat groups demonstrated significant impairments in spatial learning and memory tasks. As expected, poor performance was associated with altered NCAM1 and ERK2 expression in hippocampal tissues of SE rats. It has been reported that NCAM1 and ERK2 expressions, which are associated with neuronal remodeling and synaptic plasticity $(12,13,17)$, may be altered in the hippocampal tissues of rodent models of epilepsy $(14-16,18,19)$. Thus, this model was appropriate for investigating the effects of pharmacological interventions.

Epilepsy is a clinical syndrome caused by abnormal, highly synchronous discharges of brain neurons $(1,2)$; this condition is often accompanied by a decline in learning and memory 
Table II. Comparison of hippocampal NCAM1 mRNA and ERK2 mRNA expression in each group rats ( $\mathrm{n}=5 /$ group).

\begin{tabular}{|c|c|c|c|c|c|c|c|}
\hline \multirow[b]{2}{*}{ Group } & \multirow[b]{2}{*}{$\begin{array}{l}\text { Group } \\
\text { number }\end{array}$} & \multicolumn{3}{|c|}{ NCAM mRNA expression } & \multicolumn{3}{|c|}{ ERK2 mRNA expression } \\
\hline & & $\begin{array}{l}\text { Relative to } \beta \text {-actin } \\
\quad(\text { mean } \pm \text { SD })\end{array}$ & $\mathrm{P}$-value ${ }^{\mathrm{a}}$ & P-value ${ }^{b}$ & $\begin{array}{l}\text { Relative to } \beta \text {-actin } \\
\quad(\text { mean } \pm \mathrm{SD})\end{array}$ & P-value ${ }^{a}$ & $\mathrm{P}$-value ${ }^{\mathrm{b}}$ \\
\hline Control & 1 & $0.48 \pm 0.04$ & - & $<0.001$ & $1.33 \pm 0.16$ & - & 0.005 \\
\hline Epileptic & 2 & $0.95 \pm 0.21$ & $<0.001$ & - & $0.96 \pm 0.21$ & 0.005 & - \\
\hline Carbamazepine & 3 & $0.60 \pm 0.12$ & 0.002 & 0.005 & $0.59 \pm 0.24$ & 0.001 & 0.005 \\
\hline Oxcarbazepine & 4 & $0.66 \pm 0.03$ & $<0.001$ & 0.005 & $0.75 \pm 0.23$ & 0.001 & 0.077 \\
\hline Aniracetam & 5 & $1.18 \pm 0.17$ & $<0.001$ & 0.013 & $1.06 \pm 0.21$ & 0.011 & 0.357 \\
\hline Donepezil & 6 & $1.23 \pm 0.24$ & $<0.001$ & 0.001 & $1.27 \pm 0.18$ & 0.358 & 0.005 \\
\hline
\end{tabular}

${ }^{\mathrm{a}} \mathrm{vs}$. control group; ${ }^{\mathrm{b}} \mathrm{vs}$. epileptic group. Analysis of variance was used to compare the differences between groups. NCAM1, neural cell adhesion molecule 1; ERK2, extracellular regulated kinase 2; mRNA, messenger RNA; SD, standard deviation.

Table III. Comparison of NCAM1- and ERK2-positive neurons in the hippocampus for each group of rats (n=5/group).

\begin{tabular}{|c|c|c|c|c|c|c|c|}
\hline \multirow[b]{2}{*}{ Group } & \multirow[b]{2}{*}{$\begin{array}{l}\text { Group } \\
\text { number }\end{array}$} & \multicolumn{3}{|l|}{ NCAM1 } & \multicolumn{3}{|l|}{ ERK2 } \\
\hline & & $\begin{array}{l}\text { Number of positive neurons } \\
(\text { mean } \pm \text { SD) }\end{array}$ & P-value ${ }^{a}$ & P-value ${ }^{b}$ & $\begin{array}{l}\text { Number of positive neurons } \\
\qquad(\text { mean } \pm \mathrm{SD})\end{array}$ & P-value ${ }^{a}$ & P-value ${ }^{b}$ \\
\hline Control & 1 & $3.54 \pm 0.52$ & - & 0.001 & $22.14 \pm 1.34$ & - & 0.003 \\
\hline Epileptic & 2 & $7.92 \pm 0.46$ & 0.001 & - & $16.47 \pm 0.54$ & 0.003 & - \\
\hline Carbamazepine & 3 & $4.74 \pm 0.58$ & 0.013 & 0.008 & $10.28 \pm 1.67$ & 0.001 & 0.001 \\
\hline Oxcarbazepine & 4 & $5.42 \pm 0.82$ & 0.009 & 0.015 & $13.65 \pm 1.64$ & 0.001 & 0.010 \\
\hline Aniracetam & 5 & $9.78 \pm 0.83$ & 0.003 & 0.004 & $19.57 \pm 1.72$ & 0.008 & 0.017 \\
\hline Donepezil & 6 & $10.13 \pm 0.79$ & 0.001 & 0.002 & $20.36 \pm 1.28$ & 0.016 & 0.002 \\
\hline
\end{tabular}

${ }^{\mathrm{a}}$ Vs. control group; ${ }^{\mathrm{b}} \mathrm{vs}$. epileptic group. Analysis of variance was used to compare the differences between groups. SD, standard deviation. NCAM1, neural cell adhesion molecule 1; ERK2, extracellular regulated kinase 2.

ability $(4,5)$. With regard to treatment, the primary aim is to improve seizure control (22). However, while good clinical efficacy may be obtained with few toxic side effects, certain patients may still be resistant to a particular pharmacological agent. In addition, although seizure control may be achieved, certain agents may worsen cognitive impairments $(23,24)$. One issue underlying these differential responses to pharmacological interventions is the current limited understanding of the effects of these agents on specific cellular and molecular mechanisms.

The results of the current study revealed that, compared with control rats with good spatial learning and memory in MWM trials, saline-treated SE rats with impaired MWM trial results had significantly reduced ERK2 expression and significantly increased NCAM1 expression in hippocampal tissue at the mRNA and protein levels. That these expressions were altered in opposite directions was somewhat surprising, as NCAM expression is dependent on ERK signaling during neurogenesis, at least in vitro (20). However, these changes in expression were evaluated at 1 month after SE generation; thus, it is not known what shorter term effects may have been involved. In addition, this preliminary investigation did not determine which cell types expressed these molecules. Thus, the results may have been due to different expressions levels of NCAM1 and ERK2 on different cell types. This requires further investigation.

Nonetheless, the current results regarding intervention with different pharmacological agents revealed the same trends for NCAM1 and ERK2 expression in the hippocampal tissues of SE rats. The trends for NCAM1 and ERK2 expressions in the hippocampus (at both the mRNA and protein levels) paralleled the trends for spatial memory impairments, by either worsening (carbamazepine and oxcarbamazine) or improving (aniracetam and donepezil) MWM spatial learning/memory performance.

In general, extracellular signal-regulated protein kinases (ERKs), including ERK1 and ERK2, also called mitogen-activated protein kinases (MAPKs), are critically involved in signal transduction from cell surface receptors to the cell nucleus, and regulate numerous cell functions, including proliferation, differentiation, apoptosis and others. Thus, among other functions in the nervous system, they are essential in neurogenesis and synaptic plasticity (17). Total ERK expression and, particularly, phosphorylated forms (p-ERK) are significantly higher in the brains of patients with refractory epilepsy compared to healthy controls (18). In rodent models, ERK expression and signaling are required 
for generating epileptiform discharges $(16,19)$. Altered ERK (MAPK) expression/activity is also associated with poor MWM spatial learning task performance in rats (29). Although the expression and activity of ERKs may vary between different observation periods, it is involved with epilepsy and hippocampus-associated spatial learning/memory.

NCAMs are cell surface glycoproteins that mediate the recognition and adhesion of neural cells and are involved in axonal growth, neuronal synaptic reconstruction, loop formation and neuronal migration processes. These are important in axon regeneration and synaptic reconstruction following brain injury (30). During seizures, NCAM overexpression may lead to abnormal neuronal loop formation, which results in abnormal neuronal discharges (13). NCAMs are involved in regulating hippocampal synaptic plasticity, which is essential in regulating cognitive functions, including spatial learning and memory. Increased NCAM expression has been detected in the hippocampus in human temporal lobe epilepsy (31) and in hippocampus mossy fiber axonal sprouts in pilocarpine-induced epilepsy in rodents (32). In addition, conditional silencing of NCAM expression in the mouse hippocampus is associated with impaired spatial learning (33).

The present results from saline-treated SE rats were consistent with the general trends observed in previous reports on NCAM1 and ERK2 expression in the hippocampus of epileptic humans and rodents and their associations with impaired spatial learning. Thus, the present SE rat model was useful for, at least, evaluating the general trends for intervention with different pharmacological agents. However, these are general trends only, as the hippocampus comprises different types of cholinergic and other systems and cells, whose complex interrelationships must be taken into account (34).

Carbamazepine and oxcarbazepine are stabilizers that inactivate voltage-gated sodium channels, rendering them unavailable for subsequent opening and reducing neuron excitability. While both carbamazepine (35) and oxcarbazepine (36) are reasonably well tolerated, oxcarbazepine is less toxic over long-term usage. However, the effects of these anti-epileptic drugs on cognitive function have been a major concern, particularly carbamazepine (37). The current results indicated that these two drugs were associated with poorer spatial learning and memory compared with saline-treated SE rats (Fig. 1; Table I). In addition, relative to saline-treated SE rats, these trends were associated with reducing NCAM1 and ERK2 expression in the hippocampus; however, only the results of carbamazepine treatment were statistically significant (Figs. 2-4). The effects of these agents on worsening epilepsy-associated cognitive impairment appears to be related to their inhibiting the activities associated with NCAM1 and ERK2 expression.

Aniracetam, a so-called nootropic, is an anxiolytic agent. Although its mechanism of action is uncertain, investigations in mouse models suggest that it can act on cholinergic, dopaminergic and serotonergic systems (38). It is thought that aniracetam may be an $\alpha$-amino-3-hydroxy-5-methyl-4-i soxazolepropionic acid (AMPA) receptor agonist (glutamate analogue) that could potentially mediate against fast synaptic transmission by competing with glutamate for receptor transmission. Increased glutamate release is associated with epilepsy-related changes in the hippocampus (39). Although not statistically significant, the present results suggest that aniracetam treatment for SE rats could improve performance on spatial learning/memory tasks by increasing hippocampal tissue expression of NCAM1 and ERK2.

Donepezil is a reversible acetylcholinesterase inhibitor. By increasing the availability of acetylcholine, it is able to increase the activities of muscarinic acetylcholine receptors, which are abundant in the hippocampus (34). Thus, it may have a neuroprotective role. While donepezil has been demonstrated to be efficacious for treating cognitive impairments associated with certain neurodegenerative disorders, such as Alzheimer's disease, its effects on cognitive impairments in epilepsy are not yet firmly established (40). In the present rat SE model, however, there were significant improvements in spatial learning/memory tasks following donepezil treatment (Fig. 1; Table I), and these improvements were associated with significantly increased NCAM1 and ERK2 expression in the hippocampus at the mRNA (Figs. 2 and 3) and protein levels (Fig. 4). However, it must be considered that these effects of pharmacological intervention were for a 1 month period after SE was established.

The current preliminary investigation had several limitations. As previously noted, the specific cell types in the hippocampus expressing NCAM1 and ERK2 were not determined. In addition, only total NCAM1 and ERK2 expression, and not that of their 'activated' forms, was evaluated; in the hippocampus, epileptic activity is associated with highly polysialated NCAMs (31) and p-ERKs (18). Also not investigated were the possible dose-dependent effects of these pharmacological agents, or if shorter or longer term use may have altered the results. Finally, NCAM expression is dependent on ERK signaling during neurogenesis, at least in vitro (20); studies on NCAM1 and ERK2 co-expression in the same or different cell types also warrants investigation.

In summary, in this preliminary investigation, a rat model of SE was successfully established, and demonstrated significantly impaired hippocampus-associated spatial learning and memory. Treatment with two commonly used, first-line anticonvulsant agents (carbamazepine and oxcarbazepine) resulted in poorer performance on spatial memory tasks, whereas the nootropic aniracetam and the acetylcholinesterase inhibitor donepezil improved spatial memory task performance in SE rats. Compared to non-pharmacologically (saline) treated SE rats, these trends were paralleled by decreased (carbamazepine and oxcarbazepine) or increased (aniracetam and donepezil) expression of NCAM1 and ERK2 in the hippocampus at the mRNA and protein levels.

\section{Acknowledgements}

Financial support was provided by the Shandong Provincial Natural Science Foundation (grant no. 2009ZRB02602).

\section{References}

1. Browne TR and Holmes GL: Epilepsy. New Engl J Med 344: 1145-1151, 2001.

2. Chang BS and Lowenstein DH: Epilepsy. New Engl J Med 349: 1257-1266, 2003.

3. Scharfman HE: The neurobiology of epilepsy. Curr Neurol Neurosci Rep 7: 348-354, 2007. 
4. Hermann B and Seidenberg M: Epilepsy and cognition. Epilepsy Curr 7: 1-6, 2007.

5. Zhou JL, Shatskikh TN, Liu X and Holmes GL: Impaired single cell firing and long-term potentiation parallels memory impairment following recurrent seizures. Eur J Neurosci 25: 3667-3677, 2007

6. Liu X, Muller RU, Huang LT, Kubie JL, Rotenberg A, Rivard B, Cilio MR and Holmes GL: Seizure-induced changes in place cell physiology: Relationship to spatial memory. J Neurosci 23 : 11505-11515, 2003.

7. Parent JM, Tada E, Fike JR and Lowenstein DH: Inhibition of dentate granule cell neurogenesis with brain irradiation does not prevent seizure-induced mossy fiber synaptic reorganization in the rat. J Neurosci 19: 4508-4519, 1999.

8. Rutten A, van Albada M, Silveira DC, Cha BH, Liu X, Hu YN, Cilio MR and Holmes GL: Memory impairment following status epilepticus in immature rats: Time-course and environmental effects. Eur J Neurosci 16: 501-513, 2002.

9. Doherty P, Fazeli MS and Walsh FS: The neural cell adhesion molecule and synaptic plasticity. J Neurobiol 26: 437-446, 1995.

10. Li J, Dai G, Cheng YB, Qi X and Geng MY: Polysialylation promotes neural cell adhesion molecule-mediated cell migration in an fibroblast growth factor receptor-dependent manner, but independent of adhesion capability. Glycobiology 21: 1010-1018, 2011.

11. Wu GY, Deisseroth K and Tsien RW: Spaced stimuli stabilize MAPK pathway activation and its effects on dendritic morphology. Nat Neurosci 4: 151-158, 2001.

12. Kiss JZ and Muller D: Contribution of the neural cell adhesion molecule to neuronal and synaptic plasticity. Rev Neurosci 12 297-310, 2001

13. Seidenfaden R, Krautzer A and Hildebrandt H: The neural cell adhesion molecule NCAM regulates neurogenesis by multiple mechanisms of interaction. Neurochem Int 49: 1-11, 2006.

14. Niquet J, Jorquera I, Ben-An Y and Represa A: NCAM immunoreactivity on mossy fibers and reactive astrocytes in the hippocampus of epileptic rats. Brain Res 626: 106-116, 1993.

15. Parent JM, Yu TW, Leibowitz RT, Geschwind DH, Sloviter RS and Lowenstein DH: Dentate granule cell neurogenesis is increased by seizures and contributes to aberrant network reorganization in the adult rat hippocampus. J Neurosci 17: 3727-3738, 1997.

16. Zhao W, Bianchi R, Wang M and Wong RK: Extracellular signal-regulated kinase $1 / 2$ is required for the induction of group I metabotropic glutamate receptor-mediated epileptiform discharges. J Neurosci 24: 76-84, 2004.

17. Fukunaga $\mathrm{K}$ and Miyamoto E: Role of MAP kinase in neurons. Mol Neurobiol 16: 79-95, 1998.

18. Xi ZQ, Wang XF, He RQ, Li MW, Liu XZ, Wang LY, Zhu X, Xiao F, Sun JJ, Li JM, et al: Extracellular signal-regulated protein kinase in human intractable epilepsy. Eur J Neurol 14 865-872, 2007.

19. Houser CR, Huang CS and Peng Z: Dynamic seizure-related changes in extracellular signal-related kinase activation in a mouse model of temporal lobe epilepsy. Neuroscience 156 : 222-237, 2008

20. Mariggiò MA, Morabito C, Guarnieri S, Gentile A, Kolkova K and Fanò G: IgIII (270-280)-fragment-like H2N-DDSDEEN-COOH peptide modulates N-CAM expression via $\mathrm{Ca}^{2+}$-dependent ERK signaling during 'in vitro neurogenesis'. Peptides 29: $1486-1497,2008$

21. Rowles J and Olsen M: Perspectives on the development of antioxidant antiepileptogenic agents. Mini Rev Med Chem 12: 1015-1027, 2012

22. Shorvon S: The treatment of chronic epilepsy: A review of recent studies of clinical efficacy and side effects. Curr Opin Neurol 20 159-163, 2007.
23. Beyenburg S, Bauer $\mathrm{J}$ and Reuber $\mathrm{M}$ : New drugs for the treatment of epilepsy: A practical approach. Postgrad Med J 80: 581-587, 2004.

24. Wahab A: Difficulties in treatment and management of epilepsy and challenges in new drug development. Pharmaceuticals 3 : 2090-2110, 2010

25. Curia G, Longo D, Biagini G, Jones RS and Avoli M: The pilocarpine model of temporal lobe epilepsy. J Neurosci Methods 172: $143-157,2008$

26. Freitas RM, Oliveira AA, Sousa FCF, Vasconcelos SMM, Viana GSB and Fonteles FMM: Pathophysiology of status epilepticus induced by pilocarpine. Cent Nerv Syst Agents Med Chem 7: 11-15, 2007.

27. Racine RJ, Steingart M and McIntyre DC: Development of kindling-prone and kindling-resistant rats: Selective breeding and electrophysiological studies. Epilepsy Res 35: 183-195, 1999.

28. Hemb M, Cammarota M and Nunes ML: Effects of early malnutrition, isolation and seizures on memory and spatial learning in the developing rat. Int J Dev Neurosci 28: 303-307, 2010.

29. Shang X, Xue Y and Cai K: Expression of N-methyl-1 D-asparate receptor (NMDAR) and mitogen activated protein kinase (MAPK) in an Alzheimer disease (AD) rat model. Acta Anatomica Sinica 36: 241-245, 2005.

30. Sato K, Iwai M, Nagano I, Shoji M and Abe K: Temporal and spatial changes of highly polysialated neural cell adhesion molecule immunoreactivity in amygdala kindling development. Neurol Res 25: 79-82, 2003.

31. Mikkonen M, Soininen H, Kälviänen R, Tapiola T, Ylinen A, Vapalahti M, Paljärvi L and Pitkänen A: Remodeling of neuronal circuitries in human temporal lobe epilepsy: Increased expression of highly polysialated neural cell adhesion molecule in the hippocampus and the entorhinal cortex. Ann Neurol 44: 923-934, 1988.

32. Shan W, Yoshida M, Wu XR, Huntley GW and Colman DR: Neural (N-) cadherin, a synaptic adhesion molecule, is induced in hippocampal mossy fiber axonal sprouts by seizure. J Neurosci Res 69: 292-304, 2002.

33. Bukalo O, Fentrop N, Lee AY, Salmen B, Law JW, Wotjak CT, Schweizer M, Dityatev A and Schachner M: Conditional ablation of the neural cell adhesion molecule reduces precision of spatial learning, long-term potentiation and depression in the CA1 subfield of mouse hippocampus. J Neurosci 24: 1565-1577, 2004

34. Woolf NJ: Cholinergic systems in mammalian brain and spinal cord. Prog Neurobiol 37: 475-524, 1991.

35. Marson AG, Al-Kharusi AM, Alwaidh M, Appleton R, Baker GA, Chadwick DW, Cramp C, Cockerell OC, Cooper PN, Doughty J, et al: The SANAD study of effectiveness of carbamazepine, gabapentin, lamotrigine, oxcarbazepine, or topiramate for treatment of partial epilepsy: An unblinded randomised controlled trial. Lancet 369: 1000-10015, 2007.

36. Grant SM and Faulds D: Oxcarbazepine: A review of its pharmacology and therapeutic potential in epilepsy, trigeminal neuralgia and affective disorders. Drugs 43: 873-888, 1992.

37. Salinsky MC, Binder LM, Oken BS, Storzbach D, Aron CR and Dodrill CB: Effects of gabapentin and carbamazepine on the EEG and cognition in healthy volunteers. Epilepsia 43: 482-490, 2002.

38. Nakamura K and Kurusawa M: Anxiolytic effects of aniracetam in three different mouse models of anxiety and the underlying mechanism. Eur J Pharmacol 420: 33-43, 2001.

39. Lee TS, Mane S, Eid T, Zhao H, Lin A, Guan Z, Kim JH, Schweitzer J, King-Stevens D, Weber P, et al: Gene expression in temporal lobe epilepsy is consistent with increased release of glutamate by astrocytes. Molec Med 13: 1-13, 2007.

40. Hamberger MJ, Palmese CA, Scarmeas N, Weintraub D, Choi H and Hirsch LJ: A randomized, double-blind, placebo-controlled trial of donepezil to improve memory in epilepsy. Epilepsia 48 1283-1291, 2007. 